\title{
Vibrational and Electronic Excitations in Poly(methylphenylsilane) and Poly(diphenylsiloxane) Films: Surface Aspects
}

\section{Ana Maria Botelho do Rego and Olivier Pellegrino}

Centro de Química-F ísica Molecular, Complexo I nterdisciplinar, Instituto Superior Técnico, Avenida Rovisco Pais P-1049-001 Lisboa, Portugal

\author{
Manuel Rei Vilar* \\ Laboratoire de Dynamique, Interactions et Réactivité, Centre National de la Recherche Scientifique, 2, \\ rue Henri Dunant F-94320 Thiais, France
}

Received October 11, 2000; Revised Manuscript Received March 5, 2001

\begin{abstract}
High-resolution electron energy loss spectroscopy (HREELS) is applied to study film surfaces of two silicon-based polymers having methyl and/or phenyl rings as pendant groups: poly(diphenylsiloxane) and poly(methylphenylsilane). These spectra are compared with those obtained by infrared absorption, Raman scattering, and UV/vis absorption spectroscopy for the polymer bulk. Both vibrational and electronic spectra of these two polymers exhibit common features corresponding to the phenyl pendant group excitations. Positions of peaks appearing in HREELS vibrational spectra correspond well to infraredand Raman-active modes of the polymer bulk. Electronic losses also correspond to the electronic excited states of phenyl groups cited in the literature. Differences related to secondary emission and accumulation of relaxed el ectrons are assigned to the electronic structure of the film. The absence of a loss corresponding to the excitation of polysilane chain is related to a trapping mechanism.
\end{abstract}

\section{Introduction}

Inorganic-organic polymers (often called "inorganic polymers"), and particularly those containing silicon atoms in the backbone, constitute a field of great innovation in technological applications. ${ }^{1}$ With the exception of polysiloxanes, where fundamental studies and applications are quite advanced, ${ }^{2}$ most other inorganic polymers, and especially polysilanes, are objects of intense investigation. ${ }^{3}$ However, as far as film and surface characterization is concerned, no information is availablein the literature. Polysilanes and polysiloxanes are chainlike polymers with silicon backbone and organic substituent groups. They can therefore combine the advantages of organic polymers with those of inorganic solids-easy and cheap fabrication, mechanical strength, flexibility, and mostly resistant to corrosion and fire. ${ }^{2 b}$ Polysilanes are particularly interesting due to their $\sigma$ delocalized el ectrons along the silicon backbone giving them semiconducting properties, ${ }^{4}$ similar to conjugated organic polymers, where a $\pi$ electron delocalization exists. These polymers are expected to have multiple potential applications as photoresists, 5,6 el ectron conductive circuits, $, 7,8$ or even el ectroluminescent devices. ${ }^{9}$ They also possess very interesting mechanical properties. 10

In the past years, we have improved a method for investigating vibrational and electronic excitations in polymer surfaces by using high-resolution electron energy loss spectroscopy (HREELS) both qualitatively and quantitatively. ${ }^{11-14}$ HREELS is an adequate method for characterizing polymer surfaces ${ }^{15}$ as the vibrational modes of the chemical bonds present in this region of the film can be identified. Moreover, vibrational spectra induced by low-energy electrons can provide detailed information about the chemical composition or the

* To whom correspondence should be addressed: Tel 33149 7812 65; Fax 331497811 18, e-mail reivilar@glvt.cnrs.fr. orientation of molecules in the surface region depending on the electron mechanism involved: impact or dipolar. On the other hand, electronic excitations can also be detected by extending the spectral energy loss domain. They are mainly induced by an impact mechanism, where incident and valence el ectron exchange can occur. This exchange gives rise to features existing in UV/vis absorption spectra but even to those forbidden in optical spectroscopy by reasons of symmetry or spin conservation.

Vibrational and electronic state excitations of different polymer film surfaces using HREELS have been studied. Among them, polyethylene ${ }^{16}$ and polystyrene (PS) film surfaces were extensively analyzed using lowenergy electrons. ${ }^{17,18}$ This method is here extended to the study of films of poly(diphenyl sil oxane) (PDPS) and poly(methylphenylsilane) (PMPS), two polymers bel onging to the family of inorganic polymers with chemical structures $-\left(\mathrm{O}-\mathrm{Si}(\mathrm{Ph})_{2}-\right)_{\mathrm{n}}$ for PDPS and $-(\mathrm{Si}(\mathrm{Ph})-$ $\left.\left(\mathrm{CH}_{3}\right)-\right)_{\mathrm{n}}$ for PMPS, respectively, where $\mathrm{Ph}$ is a phenyl group. Both possess alkyl and/or phenyl functions as pendant groups. Vibrational and electronic spectra of these inorganic polymers should then present many similarities with those of the organic polymers cited above.

Most of the bands in HREELS spectra of PDPS and PMPS corresponding to electronic excitations are then expected to appear in the same domain as those observed in polystyrene due to the excitation of the pendant phenyl groups. Moreover, the $\sigma$ delocalization in the polysilane backbone also has an important consequence: the $\sigma^{*} \leftarrow \sigma$ transition occurs at a much lower excitation energy than the corresponding transition in polystyrene or in PDPS. The electronic structure of PMPS was al ready studied by one-photon and twophoton absorption spectroscopy as well as by electroabsorption by Kepler et al. ${ }^{19}$ Absorption spectra reveal an excitation to a singlet state beginning around $\mathrm{E}_{\mathrm{g}}=$ 
$3.5 \mathrm{eV}$, which also appears in electroabsorption and depends on the chain conformation. On the other hand, two-photon absorption spectra show an alternating gap, $E_{a}=4.3-4.6 \mathrm{eV}$, assigned to the second ${ }^{1} \mathrm{~A}_{\mathrm{g}}$ excitation. The same authors also performed cal culations using a Pariser-Parr-Pople (PPP) model and estimated the first triplet at 3.1 eV. Furthermore, photoluminescence studies display a broad emission centered at $2.34 \mathrm{eV}$ $(530 \mathrm{~nm})$ assigned to a $\pi^{*} \rightarrow \sigma$ transition, ${ }^{20}$ i.e., to the formation of a charge transfer state. For PDPS, an emission located in the same spectral range was detected but could not be assigned. ${ }^{21}$

In this study, PDPS and PMPS film surfaces were studied by HREELS in a spectral domain containing vibrational and electronic losses. Because of the different mechanisms governing the interaction between slow electrons and surfaces, ${ }^{22}$ sel ection rules are less restrictive. Therefore, transitions corresponding to infraredand Raman-active modes appear in vibrational HREELS spectra. Similarly, in el ectronic spectra, in addition to optically allowed transitions, forbidden transitions for symmetry or spin conservation rules are also expected to be observed. ${ }^{17,23}$

\section{Experimental Section}

Polymer films were cast from $1 \times 10^{-3} \mathrm{~g} / \mathrm{L}$ carbon tetrachloride (Chromanorm, Prolabo) solutions on sputtered platinum layers $100 \mathrm{~nm}$ thick on glass substrates. PDPS was purchased from ABCR U nited Chemical Technologies I nc. The wei ght-average molar mass ranges from 1000 to $1400 \mathrm{~g} \mathrm{~mol}^{-1}$. The chains are terminated by $\mathrm{Si}-\mathrm{OH}$ groups. PMPS was synthesized by NTT Basic Research Laboratories (Atsugi, J apan). The weight-average molar mass is $15331 \mathrm{~g} \mathrm{~mol}^{-1}$, and the polydispersity is 1.85. Terminal groups are unknown, but their relative importance is much smaller than in PDPS given the much higher molar mass. Compared to polystyrene films cast under the same conditions, the thickness is expected to be of the order of some tens of nanometers.

Infrared spectra of thin films of both polymers deposited on a silicon wafer were recorded using a Fourier transform infrared spectrometer (Magna-IR Nicolet 850) equipped with a MCT detector. The spectral domain extends from 650 to 4000 $\mathrm{cm}^{-1}$ and a resolution of $4 \mathrm{~cm}^{-1}$. Raman spectra were recorded with an XY-Dilor fitted with a CCD detector cooled with liquid nitrogen. Optical electronic spectra of films of polymers deposited on quartz substrates were acquired with a Cary 3 UV/vis spectrophotometer in a spectral range from 200 to 800 $\mathrm{nm}$.

HREELS vibrational spectra were recorded with an E LS22 Leybold-Heraeus spectrometer and HREELS electronic spectra with a Kesmodel 2000R. Both spectrometers were run under ultrahigh-vacuum conditions ( $\mathrm{P}<10^{-9} \mathrm{mbar}$ ). Incidence and analysis angles were measured relative to the normal to the surface. No charge compensation was needed, as films kept a stationary charge at the surface during the experiment as proved by the constancy of complete spectra cutoff position.

\section{Results and Discussion}

Vibrational Losses. In parts a and b of Figure 1, Raman, infrared (IR), and HREELS vibrational spectra for PMPS and PDPS films, respectively, are presented.

Main contributions to losses of HREELS spectra are presented in Table 1.

Assignments of energy losses are proposed by comparison with the corresponding infrared and Raman spectra of both polymers and according to the data published in the literature. ${ }^{24,25}$ In fact, main features appearing in HREELS spectra are composed by those found in the IR and Raman spectra of both polymers.
Table 1. Assignment of Main Features Contributing to the Losses Found in HREELS Vibrational Spectra for Polymethylphenylsilane (PMPS) and Polydiphenylsiloxane (PDPS) Films (Wavenumbers are in $\mathbf{~ c m}^{-1}$ )

\begin{tabular}{|c|c|c|}
\hline mode & PMPS & PDPS \\
\hline \multicolumn{3}{|l|}{ chain backbone } \\
\hline $\mathrm{Si}-\mathrm{Si}$ stretching & \multicolumn{2}{|l|}{$450-485$} \\
\hline $\mathrm{Si}-\mathrm{O}-\mathrm{Si}$ symmetric stretching & \multirow{3}{*}{\multicolumn{2}{|c|}{$\begin{array}{l}510-540 \\
1035-1110\end{array}$}} \\
\hline $\mathrm{Si}-\mathrm{O}-\mathrm{Si}$ asymmetric stretching & & \\
\hline end groups & & \\
\hline $\mathrm{Si}-\mathrm{OH}$ & \multicolumn{2}{|r|}{3735} \\
\hline pendant groups & & \\
\hline $\mathrm{Si}-\mathrm{C}$ (rocking) & \multirow{4}{*}{\multicolumn{2}{|c|}{$\begin{array}{l}765 \\
1440-1465 \\
2865-2885 \\
2950-2975\end{array}$}} \\
\hline aliphatic $\mathrm{CH}$ bending & & \\
\hline aliphatic $\mathrm{CH}$ sym stretching & & \\
\hline aliphatic $\mathrm{CH}$ asym stretching & & \\
\hline aromatic $\mathrm{CH}$ out-of-plane & \multirow{2}{*}{\multicolumn{2}{|c|}{$\begin{array}{c}730 \\
1030-1100\end{array}$}} \\
\hline aromatic $\mathrm{CH}$ bending in-plane & & \\
\hline aromatic CC stretching & \multicolumn{2}{|c|}{$1430-1480$} \\
\hline aromatic $\mathrm{CH}$ stretching & \multicolumn{2}{|c|}{$3030-3060$} \\
\hline
\end{tabular}

This is a general observation in HREELS spectra of different polymer surfaces. 26,27

As referred to above, electron energy losses are caused by two main mechanisms of interaction: dipole and impact. ${ }^{22}$ Due to the short interaction range in the impact mechanism-a few angstroms vs some tens of angstroms in the dipolar one-analyzed electrons are scattered by the outermost groups exposed at the polymer-vacuum interface. The sensitivity of HREELS experiment to the polymer surface is consequently referred to the extreme surface of the film, which makes of this technique as a unique tool to analyze the surface region of molecular layers. ${ }^{28}$

For monomolecular layers of small molecules deposited on well-defined metal surfaces, the traditional way to distinguish each mechanism is realized by the geometrical analysis of the different losses: dipole losses being detected in the neighborhood of the specular direction and impact losses presenting an isotropic distribution. In the case of polymer films presented here, a few hundred angstroms thick, dipolar narrow lobes of backscattered electrons do not exist, and consequently, the distinction between mechanisms is not possible by using them. Another way to identify the mechanism consists of studying the variation of the loss intensity vs the incident electron energy, $E_{p}$. In fact, differential cross sections are proportional to $I_{\text {exd }} l_{\text {elast, }}$ where $I_{\text {exc }}$ and $I_{\text {elast }}$ are the loss and the elastic peak intensities, respectively. For adsorbate on clean surfaces, dipole mechanism differential cross sections follow a decreasing power law $\mathrm{E}^{-\alpha}$ (with $0.5 \leq \alpha \leq 1$ ); contrarily, impact mechanism cross sections follow an increasing power law with $\mathrm{E}_{\mathrm{p}}$ (with $0.5 \leq \alpha \leq 1$ ). ${ }^{22} \mathrm{In}$ the case of polymer surfaces, a power law related with $E_{p}$ is also obtained, $I_{\text {exd }} I_{\text {elast }} \propto E_{p}{ }^{\alpha}$, but the $\alpha$ value varies between -1 and 1 depending on the ratio of dipolar/ impact mechanisms and the thickness of the analyzed layer. ${ }^{17}$ Since cross-section variation laws with the incident electron energy are different for each vibration mode, the relative intensity of each excitation is expected to change from spectrum to spectrum as $E_{p}$ varies. This can be observed in Figure $2 a, b$, where two sets of HREELS vibrational spectra of PMPS and PDPS films are presented for increasing $E_{p}$. These spectra were recorded in specular geometry for incidence and analysis angles of $60^{\circ}$ relative to the surface.

This means that for lower $\mathrm{E}_{\mathrm{p}}$ losses associated with strong dipole transition moments (for instance, aromatic 

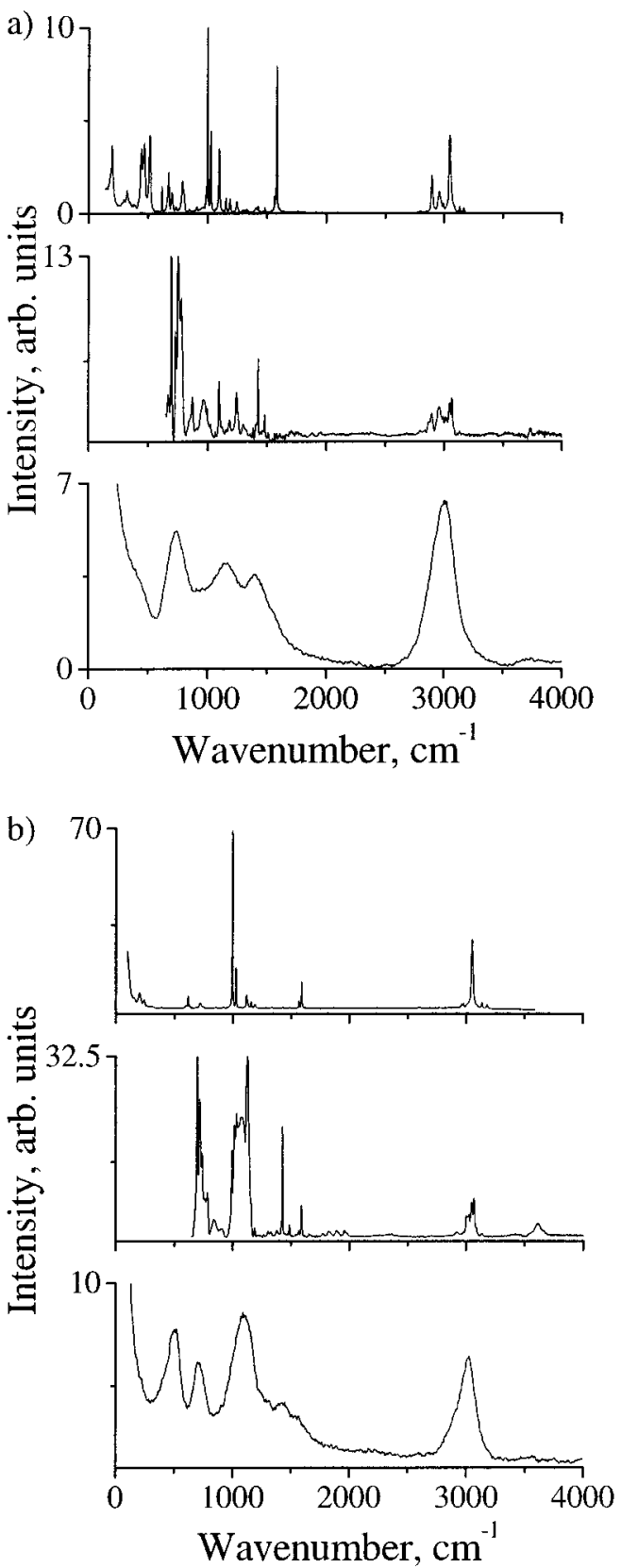

Figure 1. From bottom to top, HREELS spectrum $\left(E_{p}=1.2\right.$ $\mathrm{eV}$ in $60^{\circ}$ specular geometry), FTIRS spectrum, and Raman spectrum of (a) PMPS and (b) PDPS films.

$\mathrm{CH}$ out-of-plane deformation modes) are quite intense and decrease with $E_{p}$, whereas losses associated with impact mechanisms have low intensity and increase with $E_{p}$. One can easily notice that the intensity of most peaks increases with incident electron energy, which reveals that impact mechanisms are mainly associated with these vibrational excitations. Solely for losses assigned to the aromatic $\mathrm{C}-\mathrm{H}$ out-of-plane deformation in polysilane spectra (Figure 2a), a di polar mechanism behavior could be detected as the peak intensity decreases with the $\mathrm{E}_{\mathrm{p}}$.

Regarding the chain vibrations, PMPS has stretching and deformation modes of the silicon backbone $\mathrm{Si}-\mathrm{Si}-$ Si expected below $700 \mathrm{~cm}^{-1} .{ }^{29}$ Stretching $\mathrm{Si}-\mathrm{Si}$ modes appear in Raman spectra around $420 \mathrm{~cm}^{-1}$, and they are visible in HREELS spectra as a shoulder in the elastic peak decay. Losses corresponding to siloxane chain deformation and stretching modes of $\mathrm{Si}-\mathrm{O}$ appear

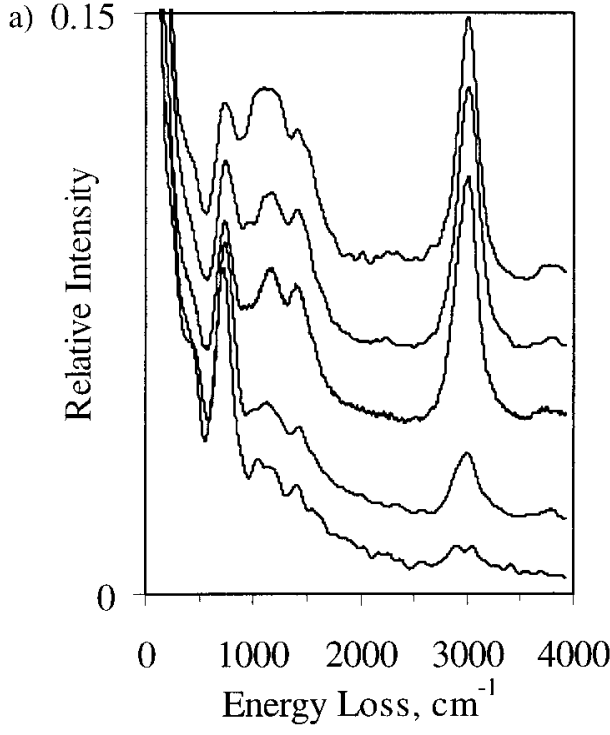

b) 0.25

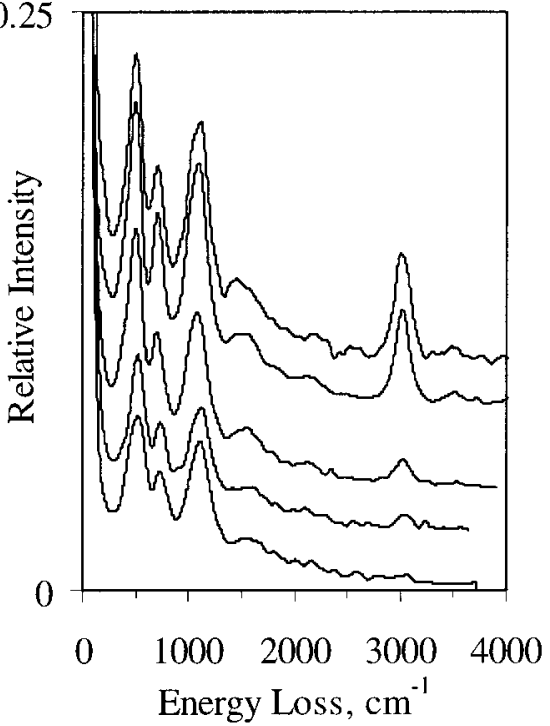

Figure 2. HREELS vibrational spectra recorded in specular geometry with angles of incidence and analysis of $60^{\circ}$ for (a) a PMPS film with electron primary energies from bottom to the top of $1.2,3.3,4.8,5.8$, and $7.8 \mathrm{eV}$ and (b) a PDPS film with the electron primary energies from bottom to the top of $1.8,2.8,4.8,5.8$, and $7.8 \mathrm{eV}$. Spectra were offset for clarity of presentation.

in HREELS spectra of PDPS (Figure 2b) around 500 and $1080 \mathrm{~cm}^{-1}$, respectively.

Despite the sensivity of HREELS to detect chain end segregation effects at the surface of the film, ${ }^{13,30}$ the presence of $\mathrm{Si}-\mathrm{OH}$ chain end groups in PDPS surface is not easily detected as the energy loss corresponding to the $\mathrm{Si}-\mathrm{OH}$ stretching mode at $3730 \mathrm{~cm}^{-1}$ falls in a spectral regi on strongly dominated by multiple scattering bands.

Electronic Excitations. In Figure 3, UV/vis photon absorption spectra are presented for the PMPS and PDPS films.

The PMPS film UV/vis absorption spectrum presents (i) an absorption band A with a threshold at $3.45 \mathrm{eV}$, which is generally assigned to the $\sigma^{*} \leftarrow \sigma$ transition cited above; ${ }^{31}$ (ii) a second feature (band B) centered around $4.4 \mathrm{eV}$, which can be partially assigned to the first singlet $\pi^{*} \leftarrow \pi$ transition of the benzene rings; and (iii) a feature $\mathrm{C}$ with a threshold around $5 \mathrm{eV}$ and 


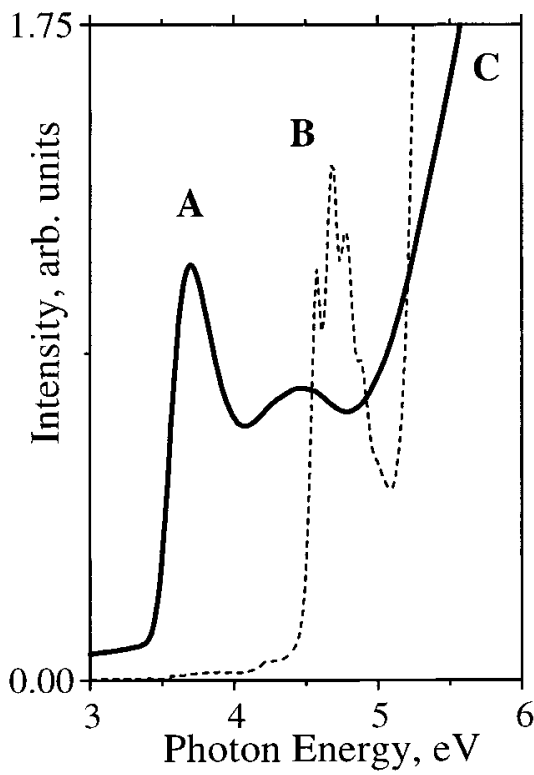

Figure 3. UV/vis absorption spectra of a PMPS film (in bold line) and a PDPS film (in dashed line).

corresponding to higher transitions of the benzene ring. ${ }^{17}$

For poly(diphenylsiloxane) films, only absorption bands $B$ and $C$ of phenyl pendant group excitations are observed.

In HREELS spectra, in addition to the relaxation of incident el ectrons by vibrational losses studied in the previous subsection, when the incident el ectron energy, $E_{p}$, is such that electronic gap $>E_{p}>$ threshold, incident electrons relax by exciting available vibrational and el ectronic transitions.

When $E_{p}>$ electronic gap, besides vibrational and el ectronic transitions, incident electrons lose energy by ionizing the medium yielding secondary el ectron emission. Consequently, HREELS spectra are composed by vibrational losses, electronic losses, and secondary el ectron emission.

To have a general overview of these results, HREELS spectra of PMPS and PDPS films are presented in Figure 4. These spectra were recorded using increasing primary energies in off-specular geometrical conditions, where the observation of impact regime is favored.

HREELS spectra of both polymers present common features corresponding to energy losses giving rise to the excitation of the phenyl group electronic (triplet and singlet) states, al ready detected in HREELS spectra of polystyrene surfaces. ${ }^{17}$ Main losses are summarized in Table 2 .

For both polymers studied here, a modification of the energy levels of the pendant group delocalized $\pi$ electrons could be expected due to bonding with silicon atoms. For poly(arylmethylsilanes), calculated energy band structure exhibits a characteristic band-edge structure due to $\sigma-\pi$ mixing between the silicon skeleton and phenyl side chains. ${ }^{32}$ In HREELS spectra, one can observe that the position of features assignable to triplet and singlet $\pi^{*} \leftarrow \pi$ transitions in both PDPS and PMPS are, within the energy accuracy, in the same energy loss as those found for polystyrene spectra. ${ }^{17}$ I n fact, HREELS electronic spectra for PDPS show a lower threshold than those found in the UV/vis spectra due to el ectron ability to excite the triplet $\pi^{*} \leftarrow \pi$ transition, forbidden in optical spectroscopy for spin conservation a)

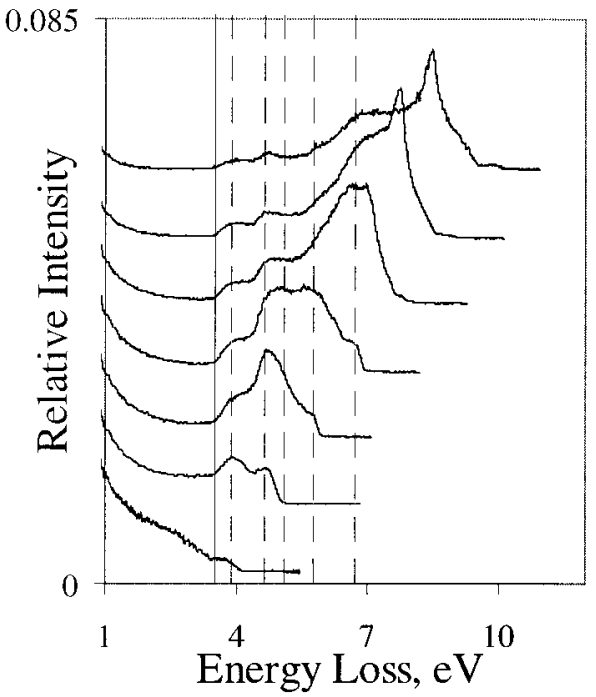

b)

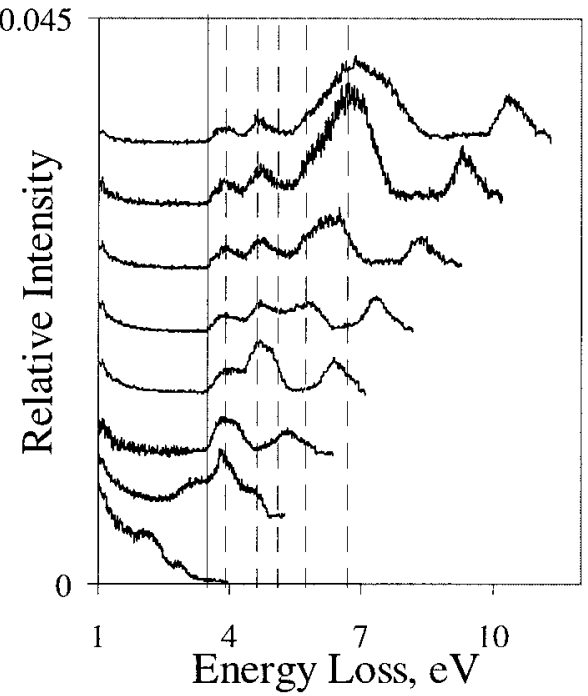

Figure 4. HREELS electronic spectra recorded for incidence and analysis angle of $60^{\circ}$ and $30^{\circ}$ using electron primary energies ranging from 3 to $11 \mathrm{eV}$ (increasing from bottom to the top by steps of $1 \mathrm{eV}$ ) for (a) a PMPS film and (b) a PDPS film. Spectra were offset for clarity of presentation. Vertical lines correspond to values to values of electronic excitations found for polystyrene surfaces (see Table 2).

Table 2. Comparison of Main Features in HREELS Spectra Corresponding to Electronic Excitations ${ }^{a}$ (Energies in $\mathrm{eV}$ ) for Benzene Compared with Those of Poly(methylphenylsilane) (PMPS) and Poly(diphenylsiloxane) (PDPS)

transition benzene and toluene (in the lit. ${ }^{a}$ ) PMPS, PDPS

$\begin{array}{lll}\mathrm{S}_{1}\left({ }^{1} \mathrm{~B}_{2 u}\right) & 4.8-5.0 & 4.7 \\ \mathrm{~S}_{2}\left({ }^{1} \mathrm{~B}_{1 u}\right) & 6.1-6.35 & 6.2 \\ \mathrm{~S}_{3}\left({ }^{1} \mathrm{E}_{1 \mathrm{u}}\right) & 6.7-7.25 & 6.7 \\ \mathrm{~T}_{1}\left({ }^{3} \mathrm{~B}_{1 u}\right) & 3.68-3.95 & 3.9 \\ \mathrm{~T}_{2}\left({ }^{3} \mathrm{E}_{1 u}\right) & 4.15-4.95 & 4.7 \\ \mathrm{~T}_{3}\left({ }^{3} \mathrm{~B}_{2 u}\right) & 4.71-5.6 & 5.6 \\ \mathrm{~T}_{4}\left({ }^{3} \mathrm{E}_{2 g}\right) & 5.96-6.02 & \end{array}$

a See, for instance, ref 17 and references therein.

reasons. In the case of PMPS, this band is situated near the expected $\sigma^{*} \leftarrow \sigma$ excitation. However, in HREELS spectra of PMPS, the relative intensity of the first and second losses (centered around 3.9 and $4.7 \mathrm{eV}$, respectively) and that of the same losses in spectra of poly(diphenylsiloxane) are roughly the same. All these observations lead to a unique conclusion, that the $\sigma^{*} \leftarrow$ 
$\sigma$ transition (band A) cited above either is not excited by electrons or is excited much less efficiently than the triplet states of benzene. These observations are quite unexpected but seem to be supported by other experimental results, which refer a strong trapping effect of the injected electrons in the conduction band of polysilanes. ${ }^{33}$ These results can be explained by a strong impact mechanism involved in the electronic excitations of the polymer. This short-range mechanism is characterized by interaction lengths of the order of a few angstroms. As an important fraction of the electron energy is involved in this process, pendant groups can constitute an efficient shielding of the chain, reducing the differential cross section of the chain excitation significantly. On the other hand, even if the electron can get an intimate contact with the chain, the strong trapping effect mentioned by Kumagai et al. ${ }^{33}$ should prevent the electron from being ejected and thence detected in the spectrum. Consequently, no losses relative to these excitations would be found in HREELS spectra.

Complete HREELS spectra of PDPS film present an isolated peak located at spectrum cutoff, always appearing at constant kinetic energy and having a slowly increasing intensity with incident el ectron energy, $E_{p}$. In contrast, ends of PMPS spectra are formed by secondary electrons emitted with a low kinetic energy, which overlap with losses corresponding to el ectronic transitions of the pendant groups.

We assign these differences to the structure of the polymer electronic band. In fact, the semiconducting property of PMPS with an el ectronic gap of about 3.5 $\mathrm{eV}$ (as observed in UV/vis absorption) is essentially due to the electronic structure of its backbone. Moreover, the existence of a narrow gap enables the appearance of secondary el ectron emission at lower $\mathrm{E}_{\mathrm{p}}$. I nstead, the $\mathrm{Si}-\mathrm{O}$ back bone confers insulating properties to PDPS as can be observed in the optical absorption spectrum, where the first excitations are those of the benzene groups appearing around $5 \mathrm{eV}$. Moreover, the large gap of PDPS makes that secondary electron emission threshold arises in HREE LS spectra for higher $\mathrm{E}_{\mathrm{p}}$ (see Figure 4). The existence of isolated peak appearing for all $E_{p}$ at the cutoff energy can be associated with a very high position of the conduction band bottom (above the vacuum level), which makes the electron affinity of the surface negative. This behavior was already observed and explained for a saturated hydrocarbon film surface. ${ }^{16}$

Losses and end of spectrum structures may be very sensitive to the geometrical arrangements in the HREELS experiment if the system has some degree of order. ${ }^{16} \mathrm{H}$ owever, since film structures were not studied by other means, no angular analysis of electronic losses or end of spectra will be presented here.

Relative Intensities as a Function of Electron Primary Energy. The relative intensity of losses as a function of the incident electron energy, $E_{p}$, is a useful tool for the comprehension of the interaction mechanism between the electron and the surface, 22,23 as al ready seen above for vibrational excitations. For electronic losses, differential cross sections usually exhibit a typical behavior of a resonance mechanism. ${ }^{17} \mathrm{~F}$ or the films studied here, differential cross sections as a function of $E_{p}$ are displayed for a vibrational loss (the aromatic $\mathrm{CH}$ stretching, at $3050 \mathrm{~cm}^{-1}$ ) and an electronic
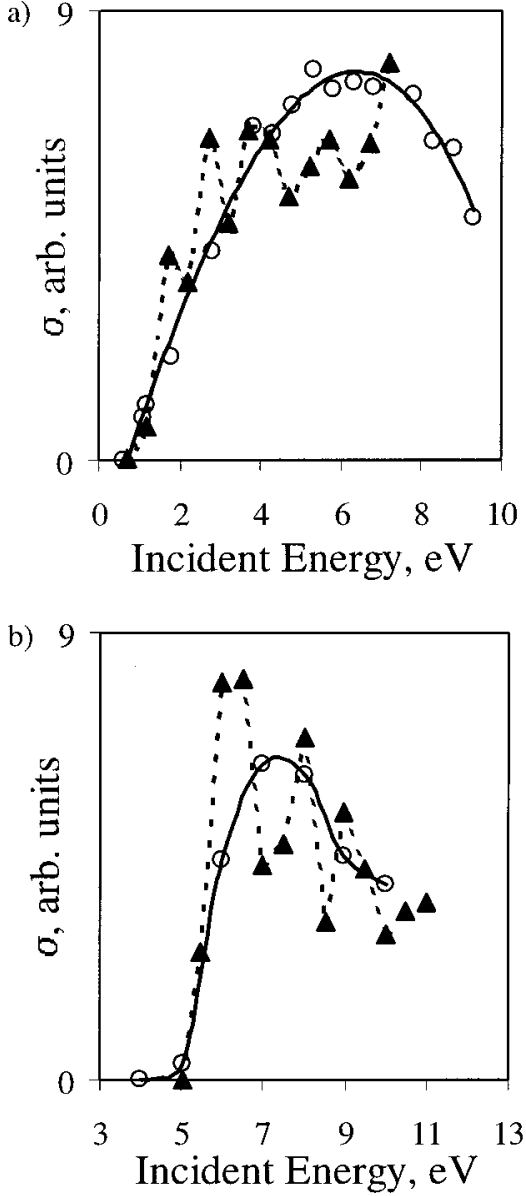

Figure 5. Differential cross section, $\sigma$, as a function of the el ectron incident energy of (a) the aromatic $\mathrm{CH}$ stretching (at $3050 \mathrm{~cm}^{-1}$ ) and (b) the electronic transition located at $\Delta \mathrm{E}=$ $4.7 \mathrm{eV}$ for PMPS (O) and PDPS ( $\mathbf{\Delta}$ ) films. Lines just serve as guides for the eye.

excitation loss (at $4.7 \mathrm{eV}$ ) in parts a and b of Figure 5 , respectively.

Measurements with the same degree of accuracy present roughly the same behavior for both films. However, the estimated differential cross sections obtained with the PDPS film vs $E_{p}$ display a greater dispersion of values than those of PMPS film, for both vibrational and electronic losses. This effect is observed for all other excitations. We assign this behavior to the fact that PDPS film is more insulating than PMPS film. Indeed, the consequently more difficult evacuation of the incoming electrons at the PDPS film favors the dispersion of differential cross section of the electron interaction process as the surface potential is more unstable.

Despite the lack of losses associated with the $\sigma^{*} \leftarrow \sigma$ transitions, HREELS spectra of PMPS evidence this transition when compared to PDPS: (i) secondary electron emission observed at lower primary energy, indicating a lower gap value; (ii) less dispersed values obtained for differential cross sections vs $E_{p}$ (cf. Figure 5), which can also be related to the semiconducting character and a better conductivity of the PMPS film.

The absence of an electron energy loss corresponding to the silicon backbone $\sigma^{*} \leftarrow \sigma$ can also be explained by a trapping process or by a screening effect of the pendant groups. Curiously, losses due to the vibrational transitions of the polysilane chain are detected. This 
observation makes the trapping effect more conceivable than the screening effect. In fact, el ectronic excitations, compared to vibrational ones, imply a higher energy loss and lower kinetic energy for the outgoing electron. Consequently, electrons producing vibrational excitations escape more easily from the trapping than those exciting electronic ones.

Nevertheless, direct observation of $\mathrm{Si}-\mathrm{Si}$ backbone transitions needs to be performed with polysilane films possessing less voluminous pendant groups. This work is in progress.

\section{Conclusions}

Both vibrational and el ectronic spectra of PMPS and PDPS exhibit common features corresponding to the excitation induced in phenyl pendant groups. In the case of vibrational spectra, IR-and/or Raman-active modes of the pendant groups and those of the corresponding chains are excited. In the electronic part of the spectra, el ectronic excitations of the phenyl groups are detected. However, in the case of PMPS, the expected energy loss corresponding to the excitation of the $\sigma^{*} \leftarrow \sigma$, characteristic of UV/vis spectrum, is not observed. This result is explained here by a screening effect of the pendant groups.

Ends of complete HREELS spectra, corresponding to secondary electrons and/or thermal electrons, are very different for the two inorganic polymers. This may be due to the different electronic band structure of the films, namely, which concerns the gap and the el ectron affinity values.

HREELS relative intensities, proportional to the corresponding cross sections of electronic losses, as a function of the incident electron energy present roughly the same behavior for both polymers, typical of a resonance mechanism. However, PDPS film presents a greater dispersion of cross-section values. This was tentatively explained by the higher insulating character of the polymer inducing a slight instability of the electrical potential of the film surface.

Acknowledgment. We thank the CNRS-ICCTI Cooperation Program between F rance and Portugal for financial support. O.P. received a post-doc fellowship (PRAXIS XXI BPD/14157/97) from the "F undação para a Ciência e Tecnologia". It is also our pleasure to thank Dr. K. Furukawa, Dr. Isaka, and Prof. N. Matsumoto from NTT Basic Research Laboratories (Atsugi, J apan) for providing the PMPS for this study and also Mr. G. Sagon from LADIR (CNRS) for the Raman spectra recording.

\section{References and Notes}

(1) Allcock, H. R. Adv. Mater. 1994, 6, 106

(2) (a) Mark, J . E.; Allcock, H. R.; West, R. Inorganic Polymers; Prentice Hall: Englewood Cliffs, NJ , 1992. (b) I bid., Chapter 4.

(3) Matsumoto, N. J pn. J . Appl. Phys. 1998, 37, 5425.

(4) Miller, R. D.; Michl, J . Chem. Rev. 1989, 89, 1359.

(5) Miller, R. D.; Wallraff, G.; Soriyakumaran, R.; Michl, J .;
Karatsu, A. J .; Mckinley, A. J .; Klingensmith K. A.; Downing, J. Polym. Eng. Sci 1989, 29, 8882.

(6) Kani, R.; Nakano, Y.; Mikoshima, S.; Hayase, S. J . Polym. Sci., Polym. Chem. 1997, 35, 2355.

(7) Kabeta, K.; Wakamatsu, S.; Sugi, S.; Imai, T. Synth. Met. 1996, 82, 201

(8) Fukushima, M.; Hamada, Y.; Tabei, E.; Aramata, M.; Mori, S.; Yamamoto, Y. Chem. Lett. 1997, 4, 347.

(9) Suzuki, H.; Meyer, H.; Hoshimo, S.; Haarer, D. J . Appl. Phys. 1995, 78, 2684.

(10) Kaito, A.; Kyotani, H.; Tanigaki, M.; Wada, M.; Yoshida, M. Polymer 2000, 41, 6395.

(11) Botel ho do Rego, A. M.; Rei Vilar, M.; Lopes da Silva, J . Heyman, M.; Schott, M. Surf. Sci. 1986, 178, 367.

(12) (a) Rei Vilar, M.; Schott, M.; Pireaux, J . J .; Grégoire, C.; Thiry, P. A.; Caudano, R.; Lapp, A.; Botel ho do Rego, A. M.; Lopes da Silva, J. Surf. Sci. 1987, 189/ 190, 927. (b) Rei Vilar, M.; Schott, M.; Pireaux, J . J .; Grégoire, C.; Caudano, R.; Lapp, A.; Botel ho do Rego, A. M.; Lopes da Silva, J . Surf. Sci. 1989, $211 / 212,782$

(13) (a) Botelho do Rego, A. M.; Rei Vilar, M.; Lopes da Silva, J. D.; Schott, M.; Petitjean, S.; J érôme, R. Macromol ecules 1993 26, 4986. (b) Rei Vilar, M.; Botelho do Rego, A. M.; Lopes da Silva, J . D.; Abel, F.; Quillet, V.; Schott, M.; Petitjean, S.; J érôme, R. Macromolecules 1994, 27, 5900.

(14) (a) Botel ho do Rego, A. M.; Pellegrino, O.; Martinho, J . M G.; Lopes da Silva, J. Langmuir 2000, 16, 2385-2388. (b) Surf. Sci., in press.

(15) Pireaux, J. J. In Surface Characterization of Advanced Polymers; Sabbatini, L., Zambonin, P. G., Eds.; VCH: Weinheim, Germany, 1993; Chapter 2.

(16) (a) Pireaux, J J .; Thiry, P. A.; Caudano, R.; Pfluger, P. J . Chem. Phys. 1986, 84, 6452. (b) Rei Vilar, M.; Schott, M.; Pfluger, P. J . Chem. Phys. 1990, 92, 5722.

(17) Botelho do Rego, A. M.; Rei Vilar, M.; Lopes da Silva, J . D. J . Electron Spectrosc. Relat. Phenom. 1997, 85, 81.

(18) Wild, S.; Kesmodel, L. L.; Apai, G. J . Phys. Chem. B 2000 104, 3179

(19) Kepler, R. G.; Soos, Z. G. Phys. Rev. B 1991, 43, 12530.

(20) Nakayama, Y.; N onoyama, S. Solid StateCommun. 1994, 92, 591.

(21) Suzuki, M.; Nakata, Y.; Nagai, H.; Okutani, T.; Kushubiki, N.; Murakami, M. Mater. Sci. Eng. 1997, B49, 172.

(22) I bach, H.; Mills, D. L. Electron Energy Loss Spectroscopy and Surface Vibrations; Academic Press: New York, 1982; Chapter 3.

(23) Kuppermann, A.; Flicker, W. M.; Mosher, A. Chem. Rev. 1979, $79,77$.

(24) (a) Socrates, G. Infrared Characteristic Group Frequencies: Tables and Charts, 2nd ed.; J ohn Wiley \& Sons: New York 1994. (b) Bellamy, L. J. The Infrared Spectra of Complex Molecules, 3rd ed.; Chapman and Hall: London, 1986; Vol. 1 , Chapter 20

(25) Bower, D. I.; Maddams, W. F. The Vibrational Spectroscopy of Polymers; Cambridge Solid State Science Series; Cambridge University Press: Cambridge, 1992.

(26) Dannetun, P.; Rei Vilar, M.; Schott, M. Thin Solid Films 1996, 286, 321.

(27) Pireaux, J . J .; Grégoire, C.; Caudano, R.; Rei Vilar, M.; Brinkhuis, R.; Schouten, J . Langmuir 1991, 7, 2433.

(28) Schreck, M.; Abraham, M.; Lehmann, A.; Schrier, H.; Goepel, W. Surf. Sci. 1992, 262, 128.

(29) Rabolt, J . F.; Hofer, D.; Miller, R. D.; Fickes, G. N. Macromolecules 1986, 19, 611 .

(30) Affrossman, S.; Hartshorne, M.; J érôme, R.; Pethrick, R. A.; Petitjean, S.; Rei Vilar, M. Macromolecules 1993, 26, 6251.

(31) I to, O.; Terazima, M.; Azumi, T.; Matsumoto, N.; Takeda, K.; Fujino, M. Macromolecules 1989, 22, 1718.

(32) Takeda, K.; Teramae, H.; Matsumoto, N.J . Am. Chem. Soc. 1986, 108, 8186.

(33) Kumagai, J .; Y oshida, H.; Koizumi, H.; Ichikawa, T. J . Phys. Chem. 1994, 98, 13117.

MA001756L 